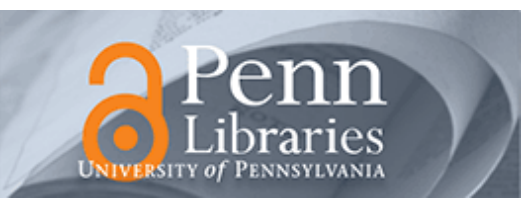

University of Pennsylvania ScholarlyCommons

March 2008

\title{
Cellular Structures of Carbon Nanotubes in a Polymer Matrix Improve Properties Relative to Composites with Dispersed
}

\section{Nanotubes}

Minfang Mu

University of Pennsylvania

Amanda M. Walker

Northwestern University

John M. Torkelson

Northwestern University

Karen I. Winey

University of Pennsylvania, winey@lrsm.upenn.edu

Follow this and additional works at: https://repository.upenn.edu/mse_papers

\section{Recommended Citation}

Mu, M., Walker, A. M., Torkelson, J. M., \& Winey, K. I. (2008). Cellular Structures of Carbon Nanotubes in a Polymer Matrix Improve Properties Relative to Composites with Dispersed Nanotubes. Retrieved from https://repository.upenn.edu/mse_papers/146

Postprint version. Published in Polymer, Volume 49, Issue 5, March 2008, pages 1332-1337.

Publisher URL: http://dx.doi.org/10.1016/j.polymer.2008.01.036

This paper is posted at ScholarlyCommons. https://repository.upenn.edu/mse_papers/146

For more information, please contact repository@pobox.upenn.edu. 


\title{
Cellular Structures of Carbon Nanotubes in a Polymer Matrix Improve Properties Relative to Composites with Dispersed Nanotubes
}

\begin{abstract}
A new processing method has been developed to combine a polymer and single wall carbon nanotubes (SWCNTs) to form electrically conductive composites with desirable rheological and mechanical properties. The process involves coating polystyrene (PS) pellets with SWCNTs and then hot pressing to make a contiguous, cellular SWCNT structure. By this method, the electrical percolation threshold decreases and the electrical conductivity increases significantly as compared to composites with a welldispersed SWCNTs. For example, a SWCNT / PS composite with $0.5 \mathrm{wt} \%$ nanotubes and made by this coated particle process (CPP) has an electrical conductivity of $\sim 3 \times 10^{-4} \mathrm{~S} / \mathrm{cm}$, while a well-dispersed composite made by a coagulation method with the same SWCNT amount has an electrical conductivity of only $\sim 10^{-8} \mathrm{~S} / \mathrm{cm}$. The rheological properties of the composite with a macroscopic cellular SWCNT structure are comparable to PS, while the well-dispersed composite exhibits a solid-like behavior, indicating that composites made by this new CPP method are more processable. In addition, the mechanical properties of the CPP-made composite decrease only slightly, as compared with PS. Relative to the common appoach of seeking better dispersion, this new fabrication method provides an important alternative means to higher electrical conductivity in SWCNT / polymer composites. Our straightforward particle coating and pressing method avoids organic solvents and is suitable for large-scale, inexpensive processing using a wide variety of polymer and nanoparticles.
\end{abstract}

\section{Keywords}

single wall carbon nanotube, polymer nanocomposite, polystyrene

\section{Comments}

Postprint version. Published in Polymer, Volume 49, Issue 5, March 2008, pages 1332-1337.

Publisher URL: http://dx.doi.org/10.1016/j.polymer.2008.01.036 


\title{
Cellular Structures of Carbon Nanotubes in a Polymer Matrix Improve Properties Relative to Composites with Dispersed Nanotubes
}

\author{
Minfang $\mathrm{Mu}^{1}$, Amanda M. Walker², John M. Torkelson² and Karen I. Winey ${ }^{*}{ }^{, 1}$ \\ ${ }^{1}$ Department of Materials Science and Engineering, University of Pennsylvania, \\ Philadelphia, PA 19104-6272, USA \\ ${ }^{2}$ Department of Chemical and Biological Engineering, Northwestern University, \\ Evanston, IL 60208-3120, USA
}

Corresponding author: tel.: 215.898.0593; fax: 215.573.2128;

e-mail winey@seas.upenn.edu 


\section{Abstract:}

A new processing method has been developed to combine a polymer and single wall carbon nanotubes (SWCNTs) to form electrically conductive composites with desirable rheological and mechanical properties. The process involves coating polystyrene (PS) pellets with SWCNTs and then hot pressing to make a contiguous, cellular SWCNT structure. By this method, the electrical percolation threshold decreases and the electrical conductivity increases significantly as compared to composites with a well-dispersed SWCNTs. For example, a SWCNT / PS composite with $0.5 \mathrm{wt} \%$ nanotubes and made by this coated particle process (CPP) has an electrical conductivity of $\sim 3 \times 10^{-4} \mathrm{~S} / \mathrm{cm}$, while a well-dispersed composite made by a coagulation method with the same SWCNT amount has an electrical conductivity of only $\sim 10^{-8} \mathrm{~S} / \mathrm{cm}$. The rheological properties of the composite with a macroscopic cellular SWCNT structure are comparable to PS, while the well-dispersed composite exhibits a solid-like behavior, indicating that composites made by this new CPP method are more processable. In addition, the mechanical properties of the CPP-made composite decrease only slightly, as compared with PS. Relative to the common appoach of seeking better dispersion, this new fabrication method provides an important alternative means to higher electrical conductivity in SWCNT / polymer composites. Our straightforward particle coating and pressing method avoids organic solvents and is suitable for large-scale, inexpensive processing using a wide variety of polymer and nanoparticles.

Keywords: single wall carbon nanotube; polymer nanocomposite; polystyrene; 


\section{Introduction}

Single wall carbon nanotubes (SWCNTs) are promising fillers for non-conductive polymers as a means to increase their electrical conductivities. Improved electrical conductivity at low SWCNT loadings is necessary for the envisioned applications of polymer nanocomposites for electromagnetic interference shielding, electrostatic protection, and electrical contacts [1-5]. To achieve high electrical conductivity, a percolated network of SWCNTs must be present, and increasing filler content above the percolation threshold results in higher electrical conductivity [6]. To lower the percolation threshold, nanotube aggregates can be exfoliated to high extents, as reported earlier [7]. Because weak van der Waals forces tend to tightly bundle SWCNTs, nanotube exfoliation requires special physical or chemical treatments: surface modification with surfactants, surface functionalization, long-time sonication, large amount of organic solvents [3, 7-10]. When exfoliation is achieved, the nanocomposites can have uniformly-distributed and highly-dispersed SWCNTs that form a percolating network above a critical concentration to give good electrical conductivity. Such SWCNT-based nanocomposites also exhibit high viscosities that hinder polymer melt processing [7, 11, 12]. Specifically, SWCNT nanocomposites with good nanotube dispersions exhibit solid-like linear viscoelastic behavior, while the matrix polymer shows liquid-like behavior under the same conditions [7, 11]. Consequently, higher temperatures and pressures will be necessary to melt process these nanocomposites, which may result in polymer degradation. Therefore, a current challenge in preparing SWCNT / polymer nanocomposites is to achieve high electrical conductivity with desirable rheological properties.

Several methods have been proposed to modify the viscoelastic properties of polymer nanocomposites while maintaining their high electrical conductivities. Plasticizers have been used in carbon black / elastomer composites to achieve improved mechanical properties, including hardness, elastic modulus and elongation at break, as compared to the elastomer [13]. Wu et al. have reported a two-phase conductive composite system in which most of the 
SWCNTs are located in the continuous polymer matrix, while a dispersed polymer phase modifies the mechanical and rheological properties of the composites [14].

Recently, a new approach to incorporate nanoscale fillers into a polymer matrix was developed using latex technology $[4,15,16]$. The latex particles (diameters 10 to $1000 \mathrm{~nm}$ ) are suspended in water with nanofillers, and upon drying, the fillers occupy the interfacial and interstitial regions between the latexes. This produces a segregated network, which exhibits a lower electrical percolation threshold, relative to composites containing uniformly-dispersed fillers. For example, the percolation threshold was 15 vol\% in a well-dispersed carbon black / poly(vinyl acetate) composite, but only 2.5 vol\% when prepared using latex particles [5]. This latex-based method that combines nanofillers with submicron-sized polymer particles has an underlying shortcoming in that the nanotubes are suspended in water using surfactants that remain in place in the final composites $[1,4,17]$. These surfactants can be detrimental to electrical conductivity [18].

This paper describes a new, straightforward method for preparing nanotube polymer composites with a continuous, macroscopic cellular structure of nanotubes that significantly increases the electrical conductivity of the composites. We demonstrate that this morphology reduces the percolation threshold for electrical conductivity, avoids the onset of solid-like rheological behavior and maintains the mechanical properties of the polymer. Our coated particle process (CPP) creates this cellular structure of SWCNTs without transforming the polymer into a latex, without adding a surfactant that hinders electrical conductivity, and without using organic solvents. Furthermore, our scalable CPP can be inexpensively applied to a wide range of polymers and fillers as a method for dramatically increasing the electrical conductivity without negatively impacting the polymer processability.

\section{Experimental section}

Single wall carbon nanotubes were synthesized by the high-pressure CO conversion (HiPCo) method at Rice University and purified with thermal oxidation, followed by $\mathrm{HCl}$ treatment [7]. All the composites in this paper used purified SWCNTs from the same batch. 
Polystyrene (PS) was purchased from the Dow Chemical Company with a weight average molecular weight of $320 \mathrm{~kg} / \mathrm{mol}$ and a polydispersity index of $\sim 2.5$, as characterized by size exclusion chromatography (SEC). The glass transition temperature $\left(\mathrm{T}_{\mathrm{g}}\right)$ of the matrix polymer is $103^{\circ} \mathrm{C}$, as detected by differential scanning calorimetry. PS arrived as rod-shaped pellets (length and diameter $\sim 3-4 \mathrm{~mm}$ ) and was either used as received or pulverized into smaller flakes, as described below.

A coated particle process (CPP) was developed to prepare SWCNT / polymer composites. Aqueous, surfactant-free suspensions of purified SWCNT were sonicated for 10 min at a concentration of $7 \mathrm{mg} / \mathrm{ml}$, mixed with PS pellets or flakes, and then sonicated for another $20 \mathrm{~min}$. This suspension was dried at $130^{\circ} \mathrm{C}$, which is higher than the $\mathrm{T}_{\mathrm{g}}$ of PS, to soften the polymer particles. While drying, the system was occasionally stirred with a spatula to quicken the water evaporation and to produce a homogenous coating of SWCNT on the surface of the softened PS pellets or flakes. The SWCNT-coated polymer particles were then held at $95^{\circ} \mathrm{C}$ overnight. The dry SWCNT-coated particles (pellets or flakes) were either compression molded at $150^{\circ} \mathrm{C}$ into large rectangular samples or processed by solid-state shear pulverization (SSSP) using a screw speed of $300 \mathrm{rpm}$ and feed rate of $\sim 50 \mathrm{~g} / \mathrm{hr}$. SSSP utilizes a modified twin-screw extruder equipped with circulating coolant to maintain all materials in the solid state. This creates high shear and compressive forces which result in repeated fragmentation and fusion of the materials.

For comparison, SWCNT / PS nanocomposites with homogeneously dispersed SWCNT bundles were prepared by our coagulation method and then compression molded. SWCNTs were suspended in N,N-dimethylformamide (DMF) at $0.1 \mathrm{mg} / \mathrm{ml}$ by 24-hr sonication. After the long time sonication, the SWCNT aggregates became small bundles with diameters of $\sim 10 \mathrm{~nm}$ and aspect ratios of $\sim 35$, as measured by atomic force microscopy (AFM). The suspension was then mixed with PS / DMF and coagulated in water [19].

For electrical conductivity measurements and reflective optical microscopy, the molded samples were cut into small pieces $\left(15 \times 10 \times 7 \mathrm{~mm}^{3}, l \times w \times h\right)$ using a diamond saw. Electrical conductivities of SWCNT / PS composites were measured on a Keiley 616 Digital Electrometer at room temperature using a two-probe method. Each composite was tested nine times and the mean and standard deviation are reported. Dynamic frequency sweeps were 
performed in the linear viscoelastic regime of the composites on a Rheometrics Solid Analyzer (RSAII) using a shear sandwich fixture and a strain of $0.5 \%$ at $210^{\circ} \mathrm{C}$ in $\mathrm{N}_{2}$. Three-point bending tests were performed on an Electromehanical Material Testing System (Instron Series 5564) at room temperature. The crosshead speed was $1.0 \mathrm{~mm} / \mathrm{min}$ and the span was $9.9 \mathrm{~mm}$, and specimen dimensions of the samples were $25 \times 5 \times 2.2 \mathrm{~mm}^{3}, l \times w \times h$. Scanning electron microscopy (SEM) was conducted on an FEI Strata DB235 Focused Ion Beam. Composites were fractured in liquid nitrogen and coated with gold before SEM imaging.

\section{Results and Discussion}

The coated particles process (CPP) involves combining polystyrene (PS) pellets with a surfactant-free aqueous suspension of SWCNT aggregates and then removing the water to form SWCNT-coated macroscopic pellets. Adhesion between the SWCNTs and polymer pellets is enhanced by quickly removing the water at a temperature above the polymer glass transition temperature. Upon compression molding the dry SWCNT-coated PS pellets, the SWCNTs form space-filling irregular polyhedrons that constitute a continuous, three-dimensional cellular structure. The size of this cellular structure is comparable to the size of the original polymer pellets. Figure 1a is an optical micrograph of a cross section of a pressed $0.5 \mathrm{wt} \%$ SWCNT-coated PS pellet composite and shows that the SWCNTs form continuous paths by being constrained to the interfacial regions between the pellets. Greater uniaxial compression during molding distorts the macroscopic SWCNT cellular structure (Figure 1b) while maintaining the cellular continuity and conductivity. (Note that pellet-pellet boundaries are not observed after molding uncoated PS pellets, because the neat PS coalesces during compression molding.)

The electrical conductivities of composites made by the CPP were measured by a two-probe method as a function of SWCNT loading (Figure 1c). A major increase in electrical conductivity occurs at $0.2-0.3 \mathrm{wt} \%$ SWCNTs, indicating the SWCNTs form an electrically conductive network that spans the sample. For example, a composite made by the CPP with 0.5 wt $\%$ SWCNTs has an electrical conductivity $\left(3 \times 10^{-4} \mathrm{~S} / \mathrm{cm}\right)$ that is $\sim 7$ orders of 
magnitude higher than PS. The level of uniaxial force used during compression molding, while enough to alter the macroscopic morphology (Figure 1a and $1 \mathrm{~b}$ ), produces composites with comparable electrical conductivities.

Figure 1c also shows the electrical conductivities for composites made by our coagulation method, first developed by Du et al. [19]. The coagulation method began with the same batch of purified SWCNTs suspended in water that was used above in the CPP. In the coagulation method, $\mathrm{N}, \mathrm{N}$-dimethylformamide (DMF) replaced the water, and the dilute SWCNT suspension $(0.1 \mathrm{mg} / \mathrm{ml})$ was sonicated for 24 hrs to produce smaller bundles. This suspension was combined with a PS / DMF solution, briefly sonicated, and then precipitated in water. Compression molding the dried precipitate forms a nanoscle, sparse network of SWCNT bundles. The electrical percolation threshold for these coagulated composites is $0.7 \mathrm{wt} \%$, which is more than double the critical concentration of the CPP-made composites. (Note that our previous work on SWCNT / PMMA composites made by coagulation found a lower threshold concentration [7], and we attribute this discrepancy to the variations in SWCNT batches and dispersion. SWCNT batches are known to vary in purity, defects, aspect ratio, and fraction of metallic SWCNT $[20,21]$. Thus, at this time it is still unreasonable to compare the absolute electrical percolation threshold of SWCNT nanocomposites from different SWCNT batches [22].) The CPP-made composites have much higher electrical conductivities $\left(10^{2}-10^{4}\right.$ times $)$ than nanocomposites made from the same SWCNTs by coagulation due to the differences in their SWCNT structures within the composites.

To better understand the cellular structure of SWCNTs in the CPP-made composites, further investigations explored the effect of polymer particle size on electrical conductivity. Solid-state shear pulverization (SSSP) uses a modified twin-screw extruder to apply high shear and compressive forces to process solid materials. Previous work with SSSP has shown improved miscibility of polymer blends and smaller dispersed phase sizes [23-25]. In this work, SSSP efficiently transforms the original millimeter-size PS pellets into polydisperse flakes of several hundred microns and also causes the molecular weight of PS to decrease slightly. Table 1 summarizes the various 0.5 wt\% SWCNT / PS composites prepared using the CPP on pellets (NTPSpel) or flakes (NTPSfla) with and without subsequent SSSP. The sample “NTPSfla1st” denotes a SWCNT / PS composite prepared by 
the CPP using PS flakes and subsequebtly subjected to one pass in the SSSP equipment. For comparison, a $0.5 \mathrm{wt} \%$ SWCNT / PS composite prepared by coagulation was also prepared (NTPScoag).

A larger initial particle size exhibits a higher electrical conductivity. In $0.5 \mathrm{wt} \%$ SWCNT composites, the electrical conductivity is $\sim 3$ orders of magnitude larger when the CPP is appled to pellets rather than flakes (Figure 2a). The PS flakes are one order of magnitude smaller than PS pellets in all dimensions, so that flakes have a surface area per volume $\sim 10$ times larger than pellets. Thus, at a fixed nanotube loading, the CPP-made cellular structure from flakes will have fewer SWCNTs per unit area at the interfaces between polymer particles, which reduces the electrical conductivity. Furthermore, the SWCNT layer is relatively inhomogeneous when the coating process occurs in water where SWCNTs are poorly dispersed. This inhomogeneity prevents some of the SWCNTs from contributing to the conductive cellular pathways, which further reduces the electrical conductivity of the composite when the areal density of SWCNTs decreases.

After a composite is formed by the CPP, the strong shear forces of SSSP disrupt the continuous SWCNT cellular network in the $0.5 \mathrm{wt} \%$ NTPSpel and NTPSfla composites. Consequenlty, after one or two passes through SSSP, the SWCNT distribution becomes more uniform. A SWCNT-rich phase and a polymer-rich phase are observed in Figures 2b and 2c; there are fewer SWCNT aggregates in NTPSpel2nd, indicating an enhanced dispersion of SWCNTs. These new morphologies exhibit better dispersion and lower electrical conductivity (Figure 2). This observation is contrary to the prevailing generalization that better SWNT dispersion increases electrical conductivity. Pulverizing the CPP-made nanocomposites disrupts the continuous, cellular network of SWCNT and thereby reduces the electrical conductivity by as much as 3 orders of magnitude.

Next we probe the rheological and mechanical properties of these composites to provide a preliminary assessment of their processability. PS and composites prepared by the CPP (0.5 wt\% SWCNTs) exhibit comparable linear viscoelastic behavior (Figure 3a). At low frequencies, the CPP-made composites have liquid-like behavior with low viscosities that aid melt fiber spinning and injection molding. Similar liquid-like rheological properties are also observed in the composites prepared from PS flakes (not shown). In contrast, a more 
homogeneous dispersion of SWCNTs (NTPScoag) produces a solid-like response, indicating that the $0.5 \mathrm{wt} \%$ SWCNTs is above the rheological percolation threshold when the nanotubes are arranged in a sparse network. The open SWCNT network produced by coagulation and indicative of good disperion leads to composites with high viscosities, which poses a substantial obstacle to traditional polymer processing methods. For example, in our work with SWCNT nanocomposite made by coagulation, we typically can melt fiber spin only below $~ 5 \mathrm{wt} \%$ SWCNTs. The cellullar structure produced by the CPP might be more amenable to typical melt processing methods.

The mechanical properties of the CPP-made nanocomposites, as measured in three point bending tests, decrease slightly with increasing SWNT loading, Figure 3b. The Young's modulus decreases only $350 \mathrm{MPa}$ as the SWCNT loading increases from 0 to $1.0 \mathrm{wt} \%$ and the yield strength drops by less than $8 \%$. The fracture surface of a CPP-made composite shows patches of PS in the SWNT-rich regions that apparently connect adjacent PS domains (Figure 3c). As the SWCNT coating becomes thicker (higher SWCNT loading for a fixed particle size), there is less interpenetration of PS across the polymer particle interface and this weakens the composites. These results suggest that during hot pressing of SWCNT-coated particles the PS chains diffuse across the interfacial SWCNT layers to reinforce the CPP-made composites without compromising the electrical integrity of the cellular SWCNT structure.

The differences in electrical, mechanical and viscoelastic properties between the CPP-made and coagulation-made SWCNT / PS composites arise from the nanotube morphologies produced by the two preparation methods. Nanocomposites made by coagulation have SWCNT networks composed of small SWCNT bundles that are arranged isotropically and can form a percolating pathway at sufficiently high concentrations. Lower percolation thresholds can be achieved in coagulation-made composites when the nanotubes are higher aspect ratio or exfoliated to a greater extent. While better exfoliation might be accomplished by extending the sonication time or introducing dispersion agents, these approaches ultimately reduce electrical conductivity by shortening the nanotubes [26], introducing structural defects in the nanotubes [27], and increasing nanotube-nanotube contact resistivities. 
In contrast, the cellular structure in the CPP-made composites is composed of SWCNT aggregates trapped at the interfacial boundaries between the polymer particles. While the short-time sonication and high SWCNT concentration in water is not sufficient to substantially exfoliate the nanotube, it does maintain the structural integrity and high electrical conductivity of SWCNTs. The three distinguishing morphological features of the CPP-made composites are as follows: nanotubes are confined to a three-dimensional cellular structure, PS reinforces the SWCNT-rich layer, and nanotubes are in aggregates rather than in the small SWCNT bundles typical of coagulation-made composites. The first and second features enable the CPP-made composites to exhibit the viscoelastic and mechanical characteristics of PS, while having a much improved electrical conductivity. The CPP-made cellular structure has a macroscopic mesh size, so that the vast majority of the PS molecules are far from the SWCNT fillers and the matrix polymer dominates the viscoelastic and mechanical behavior of these composites. In coagulation-made nanocomposites, much of the PS is within a radius of gyration of a SWCNT bundle, and this proximity imposes solid-like viscoelastic behavior on the nanocomposite. The similarity of the viscoelastic and mechanical properties between the CPP-made composites and PS promises to facilitate the commercialization of the electrically conductive nanocomposites prepared by the CPP.

This third feature noted above (large nanotube aggregates) has important consequences with respect to the electrical contact resistance between nanotube bundles. On average, the contact area between nanotube bundles in CPP-made composites will be larger than in coagulation-made composites, and this might reduce the electrical contact resistance [28]. The electrical contact resistance is very sensitive to nanoscale changes in separation; for example, it has been reported that a displacement of $0.34 \mathrm{~nm}$ increases the contact resistance by 2 orders of magnitude [29]. Therefore, the SWCNT aggregates in the CPP-made composites could have smaller contact resistance than that between the small SWCNT bundles in the coagulation-made composites, thereby increasing the electrical conductivity of CPP-made composites.

This new coated particle process is especially promising for manufacturing thermoplastics with mechanical properties comparable to the matrix polymer and substantially higher electrical conductivity. The CPP can use commercially available polymer 
pellets and aqueous suspensions of nanoparticles to produce nanocomposites with macroscopic cellular structures. This method effectively circumvents the requirements of producing a polymer latex and of introducing a surfactant to disperse the SWCNTs, although a surfactant could be used. This method avoids the use of organic solvents and is suitable for large-scale, low-cost production of polymer nanocomposites. Obviously, alterative coating methods can be incorporated into this approach including spray coating, dip coating and aerosols. Consequently, the CPP method is applicable to a vast array of thermoplastics and fillers, including carbon blacks, carbon nanofibers and metal nanowires.

\section{Conclusion:}

The coated particle process (CPP) introduces a straightforward and broadly applicable method for preparing polymer nanocomposites with a cellular structure. In brief, this method applies single wall carbon nanotubes to the surfaces of macroscopic polymer particles using aqueous suspensions and rapidly drying at temperatures higher than the $\mathrm{T}_{\mathrm{g}}$ of the polymer. The CPP-made composites confine the SWCNTs to the particle boundaries and thereby possess three-dimensional cellular structures. The SWCNT-rich interparticle layers contain both SWCNT aggregates and interpenetrating polymer, so that the viscoelastic and mechanical properties are indicative of the pure polymer. These SWCNT cellular structures provide higher electrical conductivity and lower electrical percolation threshold as compared with a nanocomposite with well-dispersed SWCNTs. This new coated particle process holds promise for manufacturing thermoplastics with mechanical properties comparable to the matrix and substantially higher electrical conductivity in a variety of polymer nanocomposites.

Acknowledgements: This research was funded by the National Science Foundation MRSEC-DMR05-20020. We gratefully acknowledge the use of SEC in Prof. S. Yang's laboratory at the University of Pennsylvania. We thank B. P. Hilman for his assistance on SEM imaging. 


\section{Reference:}

[1] Regev O; Elkati PNB; Loos J; Koning CE, Adv. Mater. 2004; 16: 248.

[2] Ou R; Gupta S; Parker CA; Gerhardt RA, J. Phys. Chem. B 2006; 110: 22365.

[3] Grossiord N; Loos J; Regev O; Koning CE, Chem. Mater. 2006; 18: 1089.

[4] Grunlan JC; Kim YS; Ziaee S; Wei X; Magid BA; Tao K, Macromol. Mater. Eng. 2006; 291: 1035.

[5] Grunlan JC; Gerberich WW; Francis LF, J. Appl. Polym. Sci. 2001; 80: 692.

[6] Moniruzzaman M; Winey KI, Macromolecules 2006; 39: 5194.

[7] Du F; Scogna RC; Zhou W; Brand S; Fisher JE; Winey KI, Macromolecules 2004; 37: 9048 .

[8] Mitchell CA; Bahr JL; Arepalli S; Tour JM; Krishnamoorti R, Macromolecules 2002; 35: 8825.

[9] Stevens JL; Huang AY; Peng H; Chiang IW; Khabashesku WN; Margrave JL, Nano Lett. 2003; 3: 331.

[10] Hobbie EK; Bauer BJ; Stephens J; Becker ML; McGuiggan P; Hudson SD; Wang H, Langmuir 2005; 21: 10284-10287.

[11] Mitchell CA; Krishnamoorti R, Macromolecules 2007; 40: 1538-1545.

[12] Yi XS; Wu G; Ma D, J. Appl. Polym. Sci. 1998; 67: 131.

[13] Huang JC; Chuang SF; Chuang TY; Grossman SJ, J. Polym. Eng. 1999; 19: 39.

[14] Wu M; Shaw L, J. Appl. Polym. Sci. 2006; 99: 477.

[15] Grossiord N; Loos J; Regev O; Koning CE, Chem. Mater. 2006; 18: 1089-1099.

[16] Hu JW; Li MW; Zhang MQ; Xiao DS; Cheng GS; Rong MZ, Macromol. Rapid Commun. 2003; 24: 889.

[17] Zhang QH; Lippits DR; Rastogi S, Macromolecules 2006; 39: 658-666.

[18] Hobbie EK; Obrzut J; Kharchenko SB; Grulke EA, J. Chem. Phys. 2006; 125: 044712.

[19] Du F; Fisher JE; Winey KI, J. Polym. Sci. Part B 2003; 41: 3333.

[20] Kiang CH; Goddard WA; Beyers R; Bethune DS, Carbon 1995; 33: 903-914. 
[21] Ebbesen TW; Lezec HJ; Hiura H; Bennett JW; Ghaemi HF; Thio T, Nature 1996; 382: 54-56.

[22] Moniruzzaman M; Winey KI, Macromolecules 2006; 39: 5194-5205.

[23] Tao Y; Kim J; Torkelson JM, Polymer 2006; 47: 6773.

[24] Lebovitz AH; Khait K; Torkelson JM, Macromolecules 2002; 35: 9716.

[25] Furgiuele N; Lebovitz AH; Khait K; Torkelson JM, Macromolecules 2000; 33: 225.

[26] Badaire S; Poulin P; Maugey M; Zakri C, Langmuir 2004; 20: 10367-10370.

[27] Ramasubramaniam R; Chen J; Liu H, Appl. Phys. Lett. 2003; 83: 2928.

[28] Buldum A; Lu JP, Phys. Rev. B 2001; 63: 161403.

[29] Fuhrer MS; Nygard J; Shih L; Forero M; Yoon YG; Mazzoni MSC; Choi HJ; Ihm J; Louie SG; Zettl A; McEuen PL, Science 2000; 288: 494-497. 
Table 1. Sample labels and characteristics of PS and 0.5 wt\% SWCNT / PS composites preapared by the coagulation method and by the coated particle process (CPP) both with and without sebsequent solid state shear pulverization (SSSP).

\begin{tabular}{lccc}
\hline Sample & $\begin{array}{c}\text { Number of } \\
\text { SSSP Passes }\end{array}$ & $\begin{array}{c}\mathbf{M}_{\mathbf{w}} \\
(\mathbf{k g} / \mathbf{m o l})\end{array}$ & $\begin{array}{c}\text { Particle } \\
\text { Shape }\end{array}$ \\
\hline PSpel & 0 & 320 & Pellet \\
NTPSpel & 0 & 320 & Pellet \\
NTPSpel1st & 1 & 320 & Flake \\
NTPSpel2nd & 2 & 320 & Flake \\
PSfla & & & \\
NTPSfla & 0 & 290 & Flake \\
NTPSfla1st & 1 & 290 & Flake \\
NTPSfla2nd & 2 & 280 & Flake \\
& & 265 & Flake \\
NTPScoag & na & 320 & na
\end{tabular}




\section{Figure captions:}

Fig. 1. Optical micrographs of the cross sections of SWCNT / PS nanocomposites prepared by the coated particle process (CPP) (a) with faceted PS domains and (b) with elongated PS domains due to higher pressures during compression molding. The lines across the polymer domains are from the diamond saw. (c) Average electrical conductivities as a function of SWCNT loading for SWCNT / PS composites made by the CPP using pellets (circles) and by the coagulation method (squares). Error bars show the standard deviation of nine measurements.

Fig. 2. (a) Electrical conductivities of $0.5 \mathrm{wt} \%$ SWCNT / PS nanocomposites prepared from SWCNT-coated PS pellets (dark) and flakes (light) without SSSP, with one pass of SSSP, and with two passes of SSSP. Optical micrographs of (b) NTPSpel1st and (c) NTPSpel2nd. Pulverization simultaneously improves the nanotube dispersion and decreases the electrical conductivity, relative to the CPP-made composites that have a cellular structure.

Fig. 3. (a) Storage shear moduli of various $0.5 \mathrm{wt} \%$ SWCNT / PS nanocomposites prepared from SWCNT-coated PS pellets, compared with PS and a composite prepared by coagulation. (b) Young's modulus and yield strength of SWCNT / PS nanocomposites prepared by the CPP from pellets as a function of SWCNT loading. (c) SEM image of the fracture surface of NTPSpel with $0.5 \mathrm{wt} \%$ SWCNT showing evidence that PS spans across the SWCNT layer. 

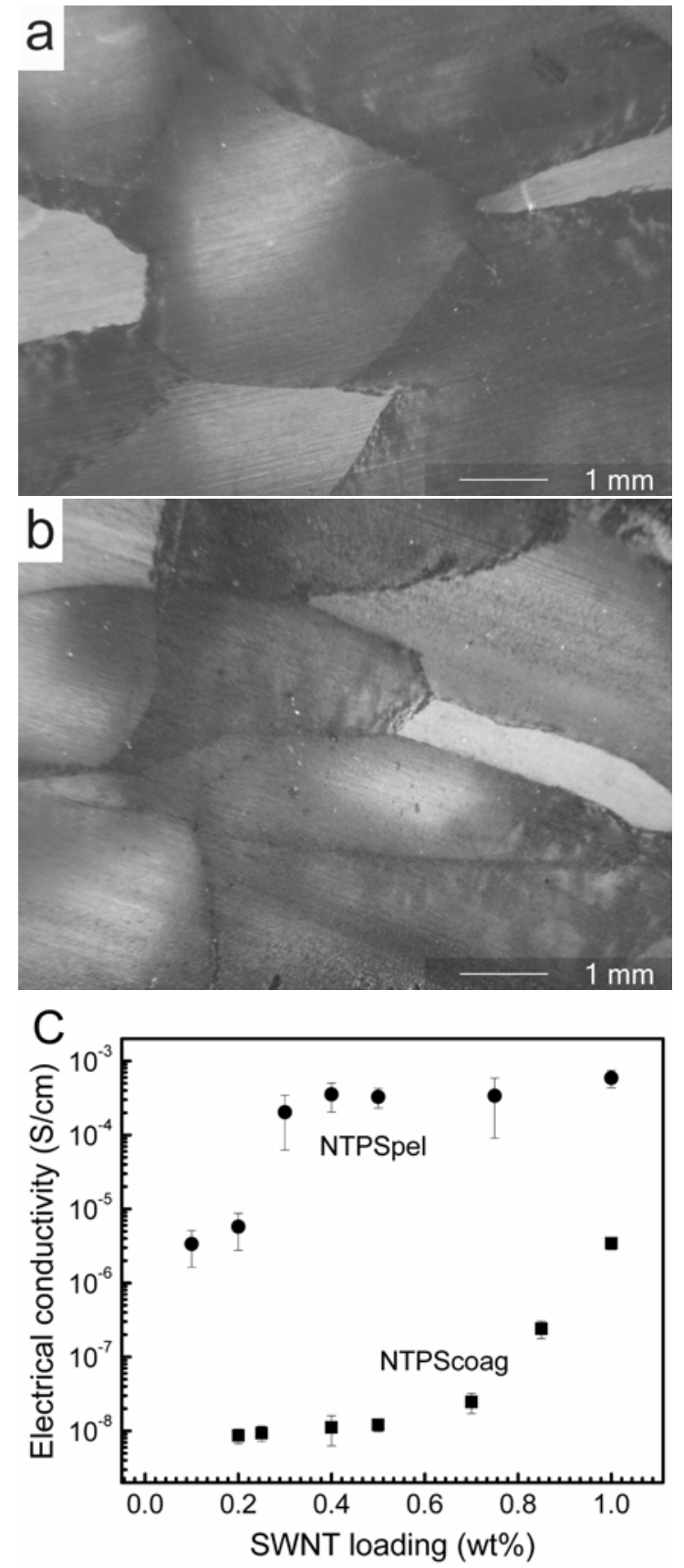

Fig. 1 

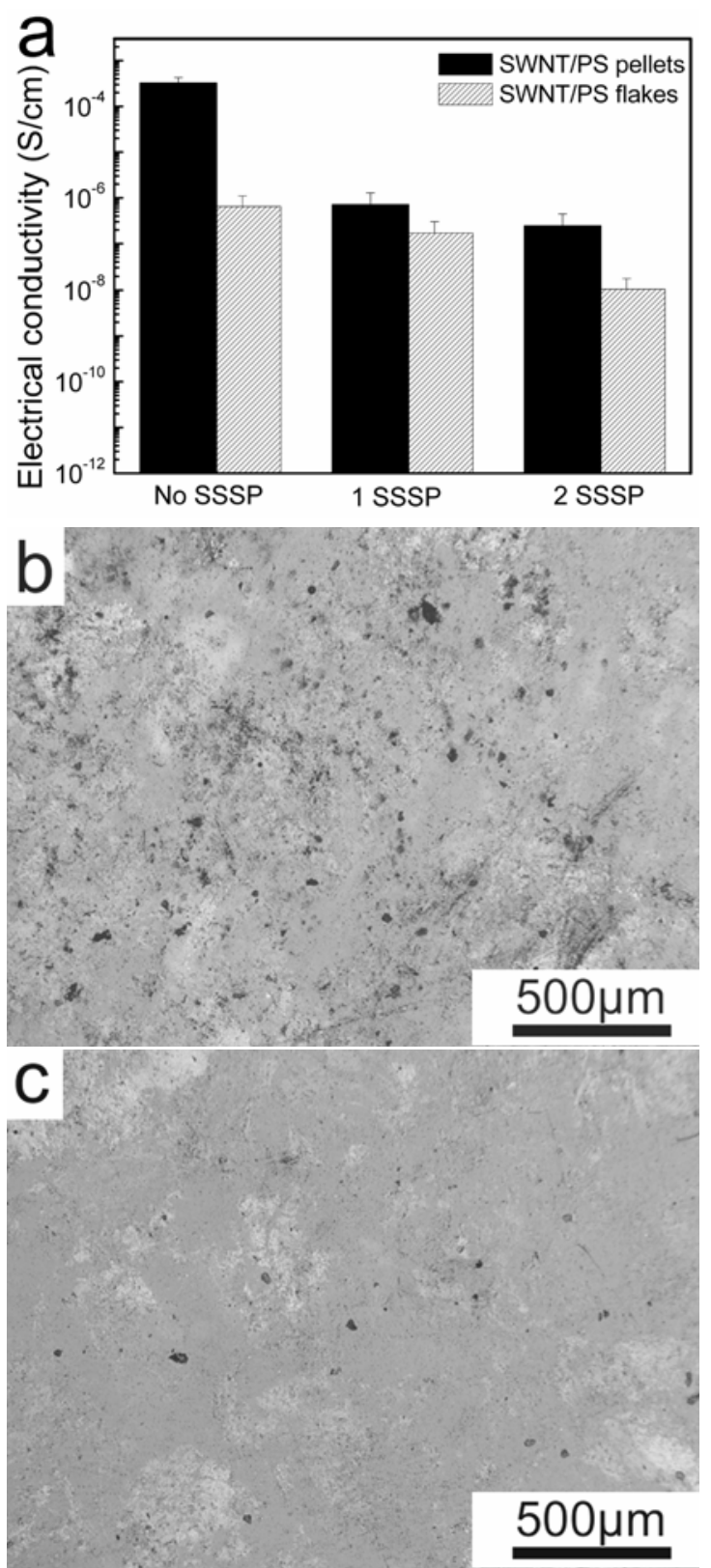

Fig. 2 

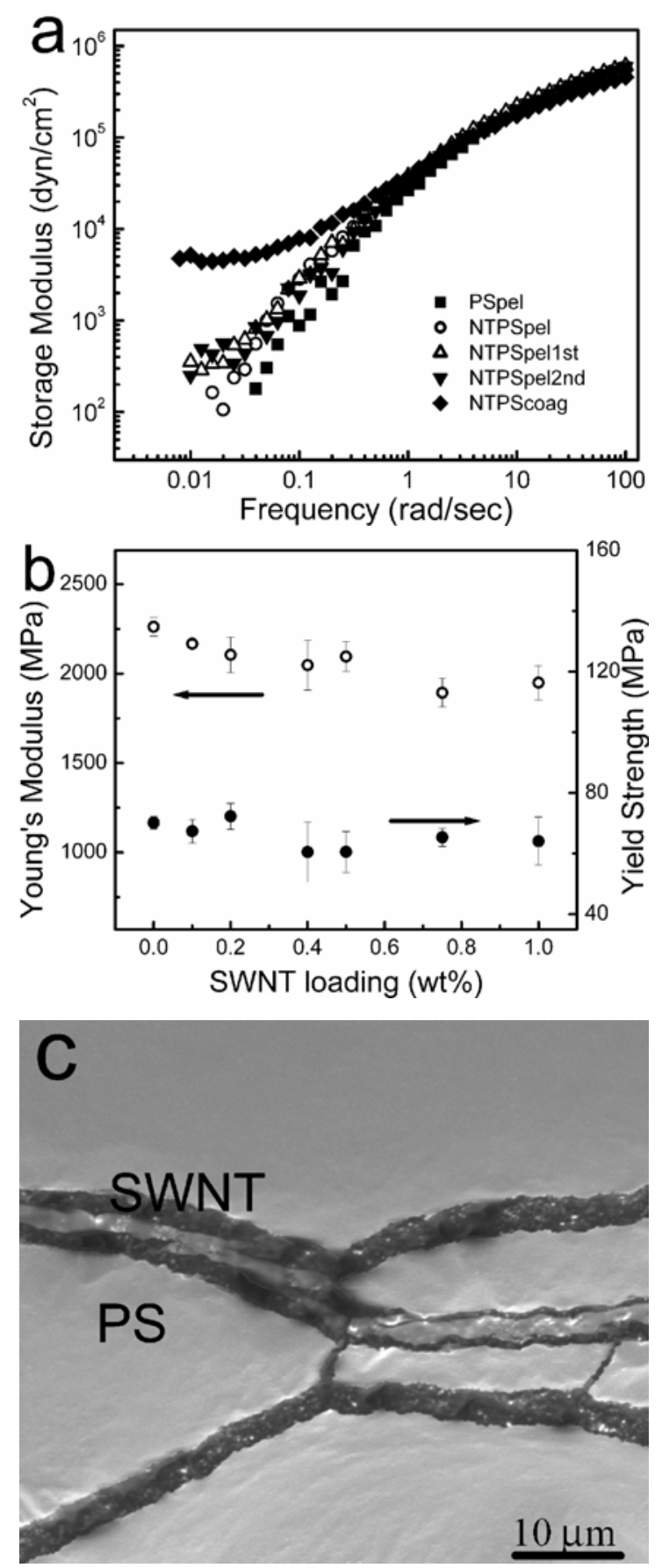

Fig. 3 